\title{
A novel hybrid microstrip/slotline transversal wide-band bandpass filter
}

\author{
Xuehui Guan ${ }^{1 a)}$, Wei Huang ${ }^{1}$, Haiwen Liu', Ye Yuan ${ }^{1}$, \\ Yande $\mathrm{Liu}^{2}$, and Zhewang $\mathrm{Ma}^{3}$ \\ ${ }^{1}$ School of Information Engineering, East China Jiaotong University, \\ Nanchang 330013, China \\ 2 School of Mechatronics Engineering, East China Jiaotong University, \\ Nanchang 330013, China \\ ${ }^{3}$ Graduate School of Science and Engineering, Saitama University, \\ Saitama 338-8570, Japan
}

a)guan@ecjtu.edu.cn

\begin{abstract}
A novel hybrid microstrip/slotline transversal bandpass filter (BPF) based on a dual-mode slotline resonator and a microstrip triple-mode resonator is proposed. Both dual-mode slotline resonator and triple mode microstrip resonator are achieved by loading a T-shaped stub to an open-loop resonator. Two resonators are set in transversal to acquire the size reduction of the filter and desired coupling between resonant modes. Based on the structure, a five-pole BPF with central frequencies of $2.75 \mathrm{GHz}$ and $0.5 \mathrm{~dB}$ bandwidth of $28 \%$ is designed. Three transmission zeros are achieved in the stopband of the filter, improving the stopband characteristics of the filter. The filter is fabricated and measured. Good agreements between measured results and simulated results verify the proposed structure well.
\end{abstract}

Keywords: transversal filter, wideband filter, hybrid structure

Classification: Microwave and millimeter wave devices, circuits, and systems

\section{References}

[1] J. L. Olvera-Cervantes, A. Corona-Chavez, D. V. B. Murthy and H. LobatoMorales: IEICE Electron. Express 6 (2009) 1143. DOI:10.1587/elex.6.1143

[2] P. Cai, Z. Ma, X. Guan, Y. Kobayashi and T. Anada: IEICE Trans. Electron. E89-C (2006) 1858. DOI:10.1093/ietele/e89-c.12.1858

[3] Y. Horii, A. Tanaka, T. Hayashi and Y. Iida: IEICE Trans. Electron. E89-C (2006) 1348. DOI:10.1093/ietele/e89-c.9.1348

[4] Y. K. Kim and S. W. Hwang: Microw. Opt. Technol. Lett. 50 (2008) 2197. DOI:10.1002/mop.23623

[5] W. Q. Che, W. J. Feng and Q. Xue: IEEE Microw. Magazine 15 (2014) 84. DOI:10.1109/MMM.2013.2296216

[6] L.-H. Hsieh and K. Chang: IEEE Trans. Microw. Theory Techn. 51 (2003) 1241. DOI:10.1109/TMTT.2003.809643

[7] S. Sun and L. Zhu: IEEE Trans. Microw. Theory Techn. 55 (2007) 2176. DOI:10.1109/TMTT.2007.906510 
[8] R. Gómez-García and J. I. Alonso: IEEE Microw. Wireless Compon. Lett. 15 (2005) 530. DOI:10.1109/LMWC.2005.852797

[9] S. Sun, L. Zhu and H. H. Tian: IEEE Microw. Wireless Compon. Lett. 18 (2008) 173. DOI:10.1109/LMWC.2008.916780

[10] R. Gómez-García: Electron. Lett. 42 (2006) 1162. DOI:10.1049/el:20062039

[11] W. J. Feng, W. Q. Che and T. F. Eibert: Electron. Lett. 47 (2011) 1330. DOI: 10.1049/el.2011.2658

[12] D. C. Rebenaque, F. Q. Pereira, J. P. García, A. A. Melcón and M. Guglielmi: IEEE Microw. Wireless Compon. Lett. 14 (2004) 475. DOI:10.1109/LMWC. 2004.834564

[13] R. Li, X. Tang and F. Xiao: IEEE Microw. Wireless Compon. Lett. 20 (2010) 328. DOI:10.1109/LMWC.2010.2047518

[14] M. Martinez-Mendoza, J. S. Gomez-Diaz, D. Canete-Rebenaque, J. L. GomezTornero and A. Alvarez-Melcon: IEEE Trans. on MTT 55 (2007) 2670. DOI:10.1109/TMTT.2007.909613

[15] S. Amari, U. Rosenberg and J. Bornemann: IEEE Trans. Microw. Theory Techn. 50 (2002) 1969. DOI:10.1109/TMTT.2002.801348

\section{Introduction}

Recently, wideband filters have been widely used in wireless systems [1, 2, 3, 4, 5, $6,7]$. A wideband bandpass filter (BPF) based on transversal signal-interference techniques was proposed in [8]. Two dissimilar transmission structures are connected in shunt and two input-to-output signal paths have been realized. In [9], a compact wideband microstrip BPF was proposed by using a transversal resonator and asymmetrical inter-digital coupled lines. Based on this principle, an ultra-wide passband can be generated between two adjacent transmission zeros by properly producing and allocating the transmission zeros. A new class of microstrip BPFs based on signal-interference principles was proposed in [10]. Transverse filter sections composed of rat-race couplers are used and UWB bandpass filtering responses is realized. In [11], a new UWB BPF with two transmission zeros close to the passband was realised by cascading a planar Marchand balun and two transmission lines with different electrical lengths. Transversal filters can also be achieved by using dissimilar resonators. In [12], two transversal microstrip resonators were used and the signal was coupled to both resonators, providing two input-to-output signal paths. In [13], a substrate integrated waveguide (SIW) transversal BPF with high selectivity was proposed by using modified doublet. Obviously, these filters are implemented by same resonators of same technology. In [14], a second-order transversal filter was implemented by combining a waveguide resonance with a microstrip resonance, which provide a new solution to wideband BPF.

In this letter, a novel hybrid microstrip/slotline transversal BPF is proposed. The filter is constructed by a dual-mode slotline resonator and a triple-mode microstrip resonator. A coupling scheme of the filter is provided and the coupling matrix is given. Compared with conventional BPF with five poles, the size of proposed filter reduces approximately eighty percents. In addition, wideband bandpass characteristics with bandwidth up to $28 \%$ has been realized. 


\section{Theory and design}

Configurations of the proposed transversal BPF is given in Fig. 1. Fig. 1a depicts the top view of the proposed filter. There is a folded stub-loaded microstrip resonator, which are directly fed by a pair of microstrip feed-lines with characteristics impedance of $50 \mathrm{ohm}$. Folded T-shaped stub is loaded to an open loop microstrip resonator, three-resonant modes are excited within the passband. Fig. $1 \mathrm{~b}$ gives the bottom view of the proposed filter, there is a T-shaped stub-loaded slotline resonator. Both resonators are directly coupled to the source and the load.

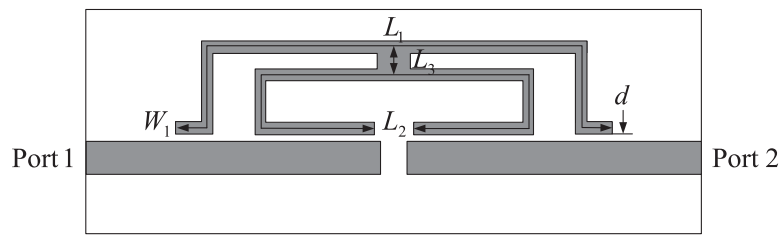

(a)

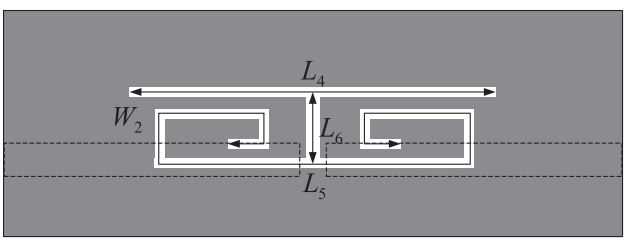

(b)

Fig. 1. Configuration of proposed transversal BPF. (a) Top view (b) Bottom view

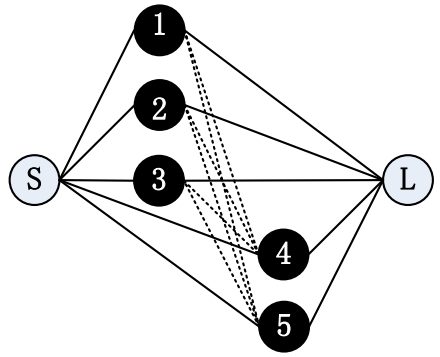

Fig. 2. The coupling scheme of proposed BPF

According to the analysis above, the coupling scheme of proposed BPF is achieved and shown in Fig. 2. Where black dots indicate the resonant mode of the resonator, and the white dots indicate source and load. Solid lines indicate the coupling between resonators and source/load, dashed lines indicate the coupling between resonant modes of resonators. Resonant modes 1, 2, 3 and 4, 5 are produced by the microstrip resonator and slotline resonator, respectively.

Therefore, corresponding coupling matrix of the proposed filter can be expressed by [15]

$$
M=\left[\begin{array}{ccccccc}
0 & M_{S 1} & M_{S 2} & M_{S 3} & M_{S 4} & M_{S 5} & M_{S L} \\
M_{1 S} & M_{11} & 0 & 0 & M_{14} & M_{15} & M_{1 L} \\
M_{2 S} & 0 & M_{22} & 0 & M_{24} & M_{25} & M_{2 L} \\
M_{3 S} & 0 & 0 & M_{33} & M_{34} & M_{35} & M_{3 L} \\
M_{4 S} & M_{41} & M_{42} & M_{43} & M_{44} & 0 & M_{4 L} \\
M_{5 S} & M_{51} & M_{52} & M_{53} & 0 & M_{55} & M_{5 L} \\
M_{L S} & M_{L 1} & M_{L 2} & M_{L 3} & M_{L 4} & M_{L 5} & 0
\end{array}\right]
$$


where $\mathrm{M}_{\mathrm{Sm}}, \mathrm{M}_{\mathrm{Ln}}$ and $\mathrm{M}_{\mathrm{mn}}(\mathrm{m}, \mathrm{n}=1 \sim 5)$ represent the coupling between source/ load and one resonant mode of the resonator, coupling between resonant modes, respectively.

Actually, the coupling between resonators and source/load are controlled by the gap and overlap length between feed line and microstrip resonator, and the coupling between feed line and slotline resonator is determined by the relation position of the resonator to the feed line.

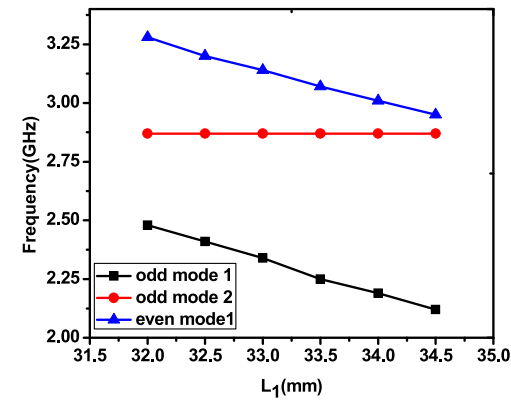

(a)

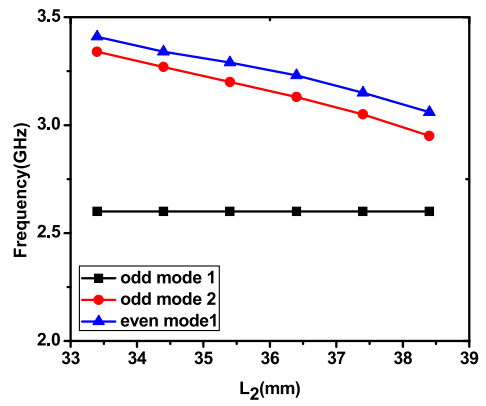

(b)

Fig. 3. The simulated frequency responses of the triple-mode resonator. (a) versus L1 and (b) versus L2

The simulated frequency responses of the triple-mode microstrip resonator versus L2 and L1 are shown in Fig. 3. When L1 increases from $31.5 \mathrm{~mm}$ to $35 \mathrm{~mm}$, odd mode 1 and even mode 1 shifts from $2.48 \mathrm{GHz}$ to 2.12 , and from $3.28 \mathrm{GHz}$ to $2.95 \mathrm{GHz}$, respectively. While the odd mode 2 remains nearly unchanged. When L2 increases from $33 \mathrm{~mm}$ to $39 \mathrm{~mm}$, odd mode 2 and even mode 1 shifts from $3.34 \mathrm{GHz}$ to $2.95 \mathrm{GHz}$, and from $3.41 \mathrm{GHz}$ to $3.06 \mathrm{GHz}$, respectively. While odd mode 1 remains stable. The simulated frequency responses of the dualmode microstrip resonator versus L4 and L5 are shown in Fig. 4. When L4 increases from $15 \mathrm{~mm}$ to $25 \mathrm{~mm}$, even mode 2 shifts from $3.32 \mathrm{GHz}$ to $2.8 \mathrm{GHz}$, and odd mode 3 shifts from $3.01 \mathrm{GHz}$ to $2.78 \mathrm{GHz}$. When L5 increases from $39.5 \mathrm{~mm}$ to $43.5 \mathrm{~mm}$, even mode 2 shifts from $3.62 \mathrm{GHz}$ to $3.01 \mathrm{GHz}$, and odd mode 3 shifts from $3.5 \mathrm{GHz}$ to $2.81 \mathrm{GHz}$. These resonant characteristics can be easily used to allocate the positions of each mode and the coupling coefficients [5].

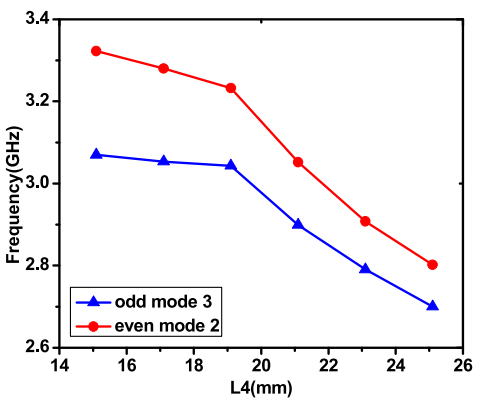

(a)

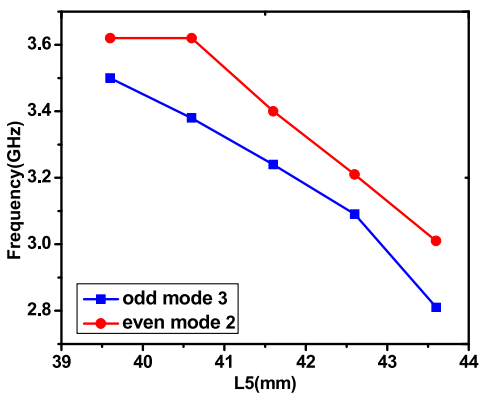

(b) 


\section{Experimental results and discussion}

In order to validate above-mentioned theory, a compact and high selectivity quintuple-mode BPF is designed and fabricated. The designed filter has a central frequency of $2.75 \mathrm{GHz}$ and a wide passband with $0.5-\mathrm{dB}$ cut-off frequency from 2.32-3.09 GHz. A substrate with a relative dielectric constant of 3.5 and a thickness of $0.8 \mathrm{~mm}$ is used in the design. Obtained parameters of the filter shown in Fig. 1 are: $\mathrm{L}=22.2 \mathrm{~mm}, \mathrm{~L} 1=33 \mathrm{~mm}, \mathrm{~L} 2=38 \mathrm{~mm}, \mathrm{~L} 3=0.9 \mathrm{~mm}, \mathrm{~L} 4=19.5 \mathrm{~mm}, \mathrm{~L} 5=$ $40.6 \mathrm{~mm}, \mathrm{~L} 6=4 \mathrm{~mm}, \mathrm{~W} 1=0.3 \mathrm{~mm}, \mathrm{~W} 2=0.5 \mathrm{~mm}, \mathrm{~d}=0.6 \mathrm{~mm}$. The filtering performance is measured by using Network Analyzer AV3926 and a comparison between EM simulated results and measured results are shown in Fig. 5. Solid lines and dotted lines indicate the simulated and measured results, respectively. Five transmission poles are clearly observed at $2.34 \mathrm{GHz}, 2.55 \mathrm{GHz}, 2.82 \mathrm{GHz}$, $3.01 \mathrm{GHz}$ and $3.09 \mathrm{GHz}$ in the passband of the filter. Three transmission zeros are generated at $1.37 \mathrm{GHz}, 2.19 \mathrm{GHz}$ and $3.75 \mathrm{GHz}$, which improve the selectivity of the filter. Apart from the insertion loss is a bit bigger that may be caused by the relatively large loss of the substrate, measured results agree well with the simulated results. The photograph of the fabricated filter is shown in Fig. 6. The overall size of the filter is about $46 \mathrm{~mm} \times 15 \mathrm{~mm}$.

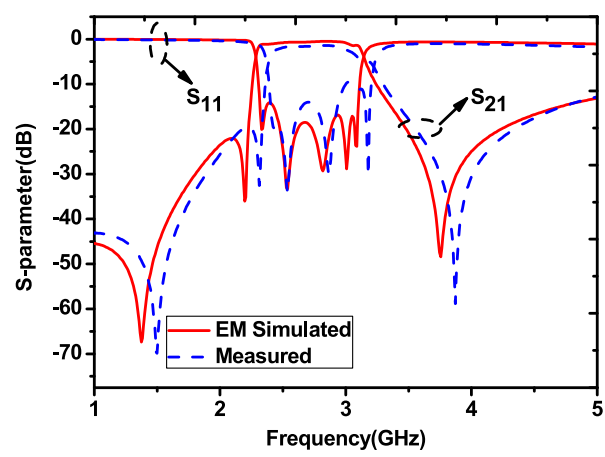

Fig. 5. Comparison between EM simulated and measured results of the proposed filter

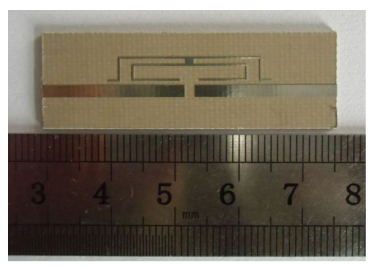

(a)

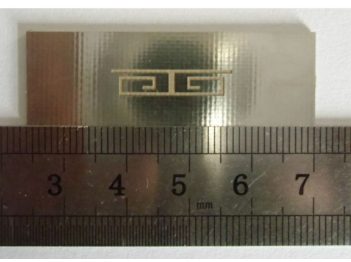

(b)

Fig. 6. Photographs of the fabricated filter. (a) Top view (b) bottom view 
established to further explain the proposed design. Three transmission zeros are realized in the stopband of the filter, which greatly improve the selectivity and attenuation of proposed filter. Measured results agree well with the simulated results, verifying the proposed structure and design methodology.

\section{Acknowledgments}

This work is supported by National Science Foundation Committee of China (No. 61161005, No. 61461020), Training Program for Young Scientists of Jiangxi Province, and Natural Science Foundation of Jiangxi Province (20152ACB21007). 